\title{
Multi-Regional Reactive Transport Due to Strong Anisotropy in Unsaturated Soils with Evolving Scales of Heterogeneity
}

\author{
Horace K. Moo-Young \\ Department of Civil and \\ Environmental Engineering \\ Lehigh University \\ 13 E. Packer Avenue \\ Bethlehem, PA 18015
}

\section{Research Objective}

Current conceptualizations of state-dependent anisotropy have failed to describe field observations, and the importance of multi-region flow in strongly anisotropic soils appears to be unrecognized. It is hypothesized that under the typical conditions of arid waste sites, when strong heterogeneity is predicted, the first order small anisotropy analysis is not rigorously applicable, and non-equilibrium mass transfer governs transport. Inter-region mass transfer is dominated by local velocity variations rather than diffusion, and access to reactive sites becomes limited. Accurate representation therefore requires an analysis to transport over evolving scales of heterogeneity and complexities to account for the interacting physical and chemical processes governing sorption in anisotropic media. In order to address these issues an integrated laboratory, field-modeling study with the following objectives is proposed.

1. Determine the scale of applicability and the limitations of the small perturbation (stochastic) approach for predicting flow and transport at arid sites with strong heterogeneity.

2. Develop an appropriately rigorous averaging approach to better quantify local scale behavior in anisotropic soils.

3. Investigate precisely a range of macroscopic flow regimes, measure accurately their three-dimensional average properties, and develop a multi-region transfer method to bridge the gap between pore-scale fluid migration and macro-scale displacement behavior under strong anisotropy.

To accomplish the stated goals and objectives, the project is investigating transport processes over evolving scales of heterogeneity and complexities using three essential components of subsurface science identified here as three unique tasks.

Task 1. Controlled Laboratory Studies.

Task 2. Controlled Field Experiments.

Task 3. Numerical Simulations Task 1 has been divided into two activities:

1) an investigation of pore-scale processes and

2) an investigation of intermediate-scale processes. Researchers at Lehigh University are focusing on part 2 of Task 1 that involves the use of a research centrifuge.

\section{Research Progress and Implications}

As of June 12, 2003 researchers have been able to derive the necessary scaling laws required to apply the Richard's equation to centrifuge modeling, and design and build a modeling box that will be utilized in the centrifuge. Centrifuge modeling allows the replication of in situ stresses at a reduced scale and provides a means of overcoming the time limitations by increasing the self-weight of infiltrating fluids. The 
centrifugal acceleration induced at the end of a rotating beam may be used to model the earth's gravitational acceleration, g. In order to correctly model stress distribution, scaling relationships need to be developed between a model and its prototype. For example a small-scale model built to a scale of $1 / \mathrm{N}$ and subjected to a centrifugal acceleration $\mathrm{Ng}$, experiences a stress distribution identical to that in a full-scale prototype subjected to the earths gravitational acceleration g. The design for the modeling box incorporates a box that is transparent to enable an observation of the propagation of the wetting front. Unstable flow is usually associated with the formation of fingers during infiltration and is therefore a factor in solute transport that needs to be understood. TDR probes will also be utilized to describe solute transport through the soils. Infiltration tests will be conducted both horizontally and vertically sequentially and not simultaneously. 